\section{PTH-113 CLINICAL RESPONSE TO INDUCTION ANTI-TNF THERAPY HAS NO EFFECT ON HAEMOGLOBIN LEVELS IN PATIENTS WITH IBD}

doi:10.1136/gutjnl-2013-304907.600

1,"T C Shepherd, ${ }^{2 P}$ Balendran, ${ }^{2} \mathrm{~J}$ Goodhand, 'E Cronin, ${ }^{1} \mathrm{~K}$ Greveson, ${ }^{2} \mathrm{E}$ Wood, ${ }^{2} \mathrm{~L}$ Marelli, 'C Murray. 'Gastroenterology, Royal Free Hospital; ' ${ }^{2}$ Gastroenterology, Homerton Hospital, London, UK

Introduction Iron deficiency anaemia (IDA), as a consequence of intestinal bleeding and inflammation, is arguably the most common complication of inflammatory bowel disease (IBD). In controlled trials, oral iron is reportedly as effective as IV iron; however, few studies have included patients with active disease where inflammation through the release of hepcidin may limit the efficacy of oral or dietary iron.

Methods To further study the relationship between inflammation and anaemia, we hypothesised that haemoglobin $(\mathrm{Hb})$ levels would rise more in anaemic patients that responded, than in patients who did not respond, to induction anti-TNF therapy. Using electronic case note review, we assessed the prevalence, severity, type and mean change in $\mathrm{Hb}(\Delta \mathrm{Hb})$ of 174 [97 Male] consecutive patients undergoing induction therapy with an anti-TNF agent [Infliximab 139: Adalimumab 35]. Anaemia was defined according to age-sex adjusted WHO criteria. Primary response was assessed at 14 weeks and defined as, at least two of; the absence of symptoms, having withdrawn from steroids, and/or a normal serum C-reactive protein. Non-response was defined as one or none of the above.

Results 89\% [156/174] patients had Crohn's disease: the mean [SD] age, age at diagnosis, and disease duration were 34 [17], 24[13], 10 [10] years respectively. Overall 49\% [85/174] patients were anaemic at initiation of ant-TNF treatment with a mean [SD] haemoglobin of 10.9 [1.3] g/dl. Only 48\% [41/85] had haematinics checked within 3 months of commencing an anti-TNF: 63\% [26/41], 31\% [13/41] had anaemia of chronic disease and $5 \%$ [2/41] were folate deficient. Overall, 37\% [31/85] of the anaemic patients were prescribed iron therapy. Considering all anaemic patients, there were no differences in the baseline $\mathrm{Hb}$ in patients who responded (11.1 [1.2] g/dl) compared with those who did not respond (10.6 [1.6] g/ $\mathrm{dl}, \mathrm{p}=0.11)$ to anti-TNF therapy. Regardless of concurrent iron therapy, there was no difference in the mean [SD] change in $\mathrm{Hb}$ $(\Delta \mathrm{Hb})$ between patients that responded to anti-TNF therapy $(0.51$ $[1.2] \mathrm{g} / \mathrm{dl})$ and those who did not $(0.47$ [1.4] $\mathrm{g} / \mathrm{dl}, \mathrm{p}=0.85)$.

Conclusion Iron deficiency anaemia is common but frequently undertreated in IBD patients receiving anti-TNF therapy. Induction anti-TNF therapy, with or without oral iron therapy, has no effect on haemoglobin levels. In patients with IDA receiving anti-TNF therapies, gastroenterologists should consider IV iron therapy.

Disclosure of Interest None Declared.

\section{PTH-114 SKIN PATHOLOGY ASSOCIATED WITH ANTI-TUMOUR NECROSIS FACTOR (ANTI-TNF) THERAPY- A SINGLE UK IBD CENTRE EXPERIENCE}

doi:10.1136/gutjnl-2013-304907.601

1."T C Shepherd, 'E Cronin, ' ${ }^{1} \mathrm{~K}$ Greveson, ${ }^{1} \mathrm{O}$ Epstein, ' $\mathrm{G}$ Erian, ${ }^{1} \mathrm{~F}$ Jaboli, ${ }^{1} \mathrm{E}$ Despott, ' $\mathrm{M}$ Hamilton, ' $\mathrm{C}$ Murray. 'Gastroenterology, Royal Free Hospital, London, UK

Introduction With the increasing use of biologic therapy in the treatment of inflammatory bowel disease (IBD) there has been a reported increase in dermatological conditions associated with therapy in patients with IBD. We carried out a prospective audit to identify the proportion of IBD patients at The Royal Free Hospital on anti-TNF therapy developing therapy related inflammatory skin pathology

Methods 141 IBD patients on anti-TNF therapy (either infliximab or adalimumab) were sent a postal questionnaire to identify patients who had experienced identifiable and associated skin conditions. The questionnaire included information regarding the body site affected, dermatology opinion and whether therapy had to be stopped. Data for infliximab and adalimumab were analysed.

Results Of 141 patients, 105 replied (71 (74\%) infliximab and 34 (74\%)adalimumab).In both groups $32 \%$ of patients described new skin complaints attributable to anti-TNF therapy $(n=23$ in infliximab group, $n=11$ in adalimumab group). Sites of skin inflammation were common to both groups; face (29\%), trunk (21\%), legs $(14 \%)$ and arms (14\%). Combined data showed only $44 \%$ of patients were reviewed by a dermatologist and received a formal diagnosis. No patients on adalimumab stopped treatment, while 3 stopped therapy in the infliximab group ( $9 \%$ overall).

Conclusion Although IBD is itself associated with skin pathology, recent studies have demonstrated that patients on anti-TNF therapy develop inflammation of the skin (1), and our data support the concept that paradoxical skin inflammation related to anti-TNF therapy is a class effect. [c1] In our cohort few patients had to stop therapy which is similar to some ${ }^{(1)}$, but not all reported studies ${ }^{(2,3)}$. Less than half of affected patients received consultant dermatological review.

responded. [c1]Can't just give a figure 'if' they had

Disclosure of Interest None Declared.

\section{REFERENCES}

1. Cleynen I, Vermeire S. Paradoxical inflammation induced by anti-TNF agents in patients with IBD. Nat Rev Gastroenterol Hepatol. 2012 Sep; 9(9):496-503.

2. Cullen, G., Kroshinsky, D., Cheifetz, A. S. \& Korzenik, J. R. Psoriasis associated with anti-tumour necrosis factor therapy in inflammatory bowel disease: a new series and a review of 120cases from the literature. Aliment. Pharmacol. Ther. 34, 13181327 (2011).

3. Baumgart, D. C., Grittner, U., Steingraber, A., Azzaro, M. \& Philipp, S. Frequency, phenotype, outcome, and therapeutic impact of skin reactions following initiation of adalimumab therapy: experience from a consecutive cohort of inflammatory bowel disease patients. Inflamm. Bowel Dis. 17, 2512-2520 (2011).

\section{PTH-115 OUTCOME OF ULCERATIVE COLITIS PATIENTS THAT HAVE FAILED CONVENTIONAL THIOPURINE THERAPY; ALLOPURINOL CO-THERAPY AND BEYOND}

doi:10.1136/gutjnl-2013-304907.602

1.'T Hollingworth, 'H Johnson, 'R Basuroy, 'S Mclaughlin, 'S Weaver. 'Gastroenterology, Royal Bournemouth Hospital, Bournemouth, UK

Introduction Treatments available for Ulcerative Colitis (UC) are limited in those who fail thiopurines. British society of gastroenterology guidelines suggest the use of methotrexate (MTX) or colectomy in this group as biologics are not funded in UC in England or Wales. Clinical treatments used in our unit include low dose thiopurine and allopurinol co-therapy (LDTA) and topical arsenic. We report our experience of patients who have failed standard dose thiopurines and their subsequent treatment and clinical outcomes.

Methods UC patients who failed conventional thiopurine therapy were identified from our prospective inflammatory bowel disease database. Reasons for failure, disease extent, length of follow-up and subsequent treatment pathway were identified. Data from patients with $<3$ months follow-up was disregarded.

Results 20 patients were identified, 12 initially started LDTA and 10 started MTX (2 patients received LDTA and then MTX). Mean age was 47 (range: 22-60yrs), 15 were male. 8 had pancolitis, 9 left sided UC and 4 proctitis. Reasons for failure of conventional dose thiopurine were; Hepatitis $(n=5)$, therapeutic 6-thioguanine levels without clinical remission $(n=11)$, intolerable side effects $(n=3)$, dyserythropoeisis $(n=1)$ and gout $(n=1)$. 